\title{
An ethnobotanical survey of indigenous flora for treating tuberculosis and other respiratory diseases in Niger State, Nigeria.
}

\author{
Abdullahi Mann ${ }^{\mathrm{a} *}$, J.O. Amupitan ${ }^{\mathrm{b}}$, A.O. Oyewale ${ }^{\mathrm{b}}$, \\ J.I. Okogun ${ }^{\mathrm{c}}$ and Ibrahim, Kolo ${ }^{\mathrm{d}}$. \\ ${ }^{a}$ Department of Science Laboratory Technology, Federal Polytechnic, P.M.B. 55, Bida, Niger State, Nigeria. \\ ${ }^{b}$ Department of Chemistry, Ahmadu Bello University Zaria, Kaduna State, Nigeria. \\ 'Department of Medicinal Plant Research and Traditional Medicine, National Institute for Pharmaceutical \\ Research and Development (NIPRD), P.M.B. 21, Garki - Abuja. \\ ${ }^{d}$ Department of Microbiology, Human Virology and Biotechnology, National Institute for Pharmaceutical \\ Research and Development (NIPRD), P.M.B.21, Garki Abuja.
}

\begin{abstract}
An ethnobotanical survey of medicinal plants used locally for treating tuberculosis (TB) and other respiratory diseases was conducted from November 2004 to March 2005 in Niger State-Nigeria. The survey was aimed at identifying plants used in traditional medicine for treating TB and other pulmonary ailments in Niger State. Tuberculosis, cough, asthma, hemoptysis and bronchitis were the major ailments treated in this survey. The interviews were conducted among the traditional medical practitioners and herbal traders in both urban and rural areas of the state. It was observed that the use of traditional medicine for treating respiratory diseases was predominantly widespread in Niger State among all the ethnic groups and intertwines with orthodox medication. A total of 95 medicinal plants belonging to 48 families used in 86 herbal recipes were surveyed. In many recipes, admixtures of plants were used. A total of 12 different kinds of respiratory ailments were encountered in this study. The index of performance (Ip) was calculated for each plant species. The plants with high Ip are often confirmed to possess biologically active chemical substances in the literature. A good number of plants identified in this survey for treating tuberculosis with $(I p>0)$ have been reported to possess significant anti-tubercular activity. Therefore, further screening of those plants with high performance indices as sources of 'lead' for antitubercular agents is highly recommended.
\end{abstract}

Keywords: Medicinal plants; Herbal recipes; Respiratory diseases; Tuberculosis; Index performance (Ip); Antitubercular activity

\section{Introduction}

The early man knew that some plant extracts could treat diseases. Galen said that there was no disease, which plants could not cure [1]. Primitive man probably found the therapeutic property of herbs by trial and error or by accident. The 
knowledge so obtained has been carried from generation to generation by oral tradition with almost no written form of record. Ethnobotanical studies carried out throughout Africa confirmed that local plants are the main constituents of traditional African medicines [2-5].

The growing interest in the studies of medicinal plants especially the tropical plants of Africa, stemmed from the fact that many inhabitants of developing countries, Nigeria inclusive lack sufficient westernized medical facilities (that is, in terms of the development of drugs, problems of cost and transportation of drugs) [6]. Moreover, traditional medicine remains the readily alternative source of medicaments for 80 percent of the people because of its affordability, reliability (in terms of availability) and accessibility [7,8]; therefore, attempts are made to validate the efficacy of these remedies proclaimed for better health-care delivery. With the current trends of the high depletion of tropical forest resources and little known about most of the world's plant species especially African tropical rainforest floras, there is need to survey and document the medicinal flora of indigenous communities.

Worldwide, tuberculosis and related respiratory diseases have continued to be one of the most dreaded infections in the world particularly in Africa since the emergence of HIV/AIDS. In particular, TB statistics are even more frightening. It is estimated that one third of the world's population is infected with TB, with about 8 million new cases annually [9]. Of these, 3 million die annually, more deaths than caused by malaria or any other single infectious disease. TB is the leading killer of youths, women, and AIDS patients in the world [10]. The situation is exacerbated by impediments such as multidrug resistant-TB, non-compliance with the conventional treatment regimens due to their cost and cumbersome nature and selfmedication by most infected persons in the society. Several indigenous communities around the world use herbal medicine for the traditional treatment of TB and other respiratory ailments [11-20].This observation confirms the universal application of plants as curative agents for TB and other respiratory diseases.

Generally, certain areas of vegetation (Savannah and Guinean forest) are rich in species and types of environments to be used to search for natural products, whose choice can be based on ethnobotanical and chemotaxonomic studies. Nigeria's diverse flora offers a wide spectrum of unique medicinal plants $[3,21,22]$. Niger State is one of the thirty-six states of Nigeria. The state is centrally located in the middle belt of Nigeria. It is made up of 25 Local Government Areas (LGA).It is bordered by six states including Federal Capital Territory. The state also shares a boundary with the Republic of Benin in Agwara LGA. The state has a population of above 3 million and a land area of 80,000 square kilometres, making it one of the 
largest states in Nigeria with approximately 10 percent of the total land mass of Nigeria. The state is naturally endowed with both savannah and tropical rainforest vegetations. There are three major ethnic groups in Niger State that is, the Nupes, the Hausas and the Gwaris. The Nupe together with other groups such as Kakanda, Dibo, Ganagana, occupy the southern geographical area with Nupe language being predominantly spoken. The Gwari as well as the Pangu, Kamuku, Kadara and Koro reside in the eastern geographical zone where Gwari is the indigenous language spoken by majority of the people. The Hausa and other ethnic groups like Kambari, Dukawa, Achifawa and Ingwai consist of the northern zone and Hausa is spoken and understood by the majority of the indigenous inhabitants of this zone. The people of Niger State are predominantly agrarian by nature, farming being their major occupation. These tribes have long standing traditions of herbal medicine that dates back to the pre-colonial era because it is interwoven with farming practices. Currently, traditional herbal medicine is practiced together with modern conventional medicine. Patients often combine herbal and orthodox medications. Nupe, Hausa and Gwari communities of Niger State are among the Nigerian communities that presently, to a large extent depend solely on the traditional herbal medicine for the treatment of TB and other respiratory diseases.

Several scientific investigations of medicinal plants have been initiated in many countries because of their contributions to health care [2, 7, 21-23]. Plants which are observed to be efficacious [24-27] and frequently prescribed may contain compounds that are potential drug candidates or drug leads and could rightly be recommended for further examination [28, 29]. It is also known that plant sampling based on ethnobotanical survey approach showed greater percentage yield of bioactive useful medicinal compounds than other methods [30,31]. It is against this background, that ethnobotanical studies on the medicinal properties of Nigerian Nupeland plants have been carried out [3]. However, only a relatively small number of medicinal plants have been thoroughly investigated for respiratory diseases, more so as the survey of the ethnomedicine for tuberculosis and other respiratory diseases in Niger State has not been reported. The dearth of this information coupled with the ever-increasing need for new anti-TB agents from natural products to curb multidrug resistant $\mathrm{TB}$ and other respiratory diseases necessitated this study.

\section{Materials and methods}

\section{Study}

The method described by Betti (2004) was used in this study [12]. It consists of gathering data on the commonly used medicinal plants in a given area. The originality and the strength of this method is derived from its qualitative and quantitative approach. Following this method, the data for this study were obtained 
from oral interviews and questionnaires administered to the indigenous people from November 2004 to March 2005 in Niger State. To ensure effective survey coverage of common herbal recipes among the three major tribes, the three geographical regions of the state were used and identified as zones A, B and C.

The interviews were done in both urban and rural areas of the zones. The interviewers principally made use of the members of the existing herbal association called Nigerian Association of Traditional Medical Practitioners and in some cases herbal traders in the urban markets of Bida, Minna and Kontagora. A concise questionnaire (Appendix 1) was designed and prepared to obtain the most precise ethnobotanical data. The team also enlisted the support of the community leaders such as the Emirs, Village-heads, and Ward-heads in addition to the members of well-organized herbal association. The local names of the plants were recorded as much as possible in five Nigerian languages (Table 1) on the basis of the fact that Hausa, Yoruba and Igbo are the three most widely spoken languages in Nigeria and at the same time these groups of people are found in all parts of the state. Nupe, Hausa and Gwari, are the indigenous languages spoken in the area of study.

\section{Identification of plant species}

Efforts were made to immediately collect plants mentioned by the herbal informants for proper identification. Identification was done in the Department of Biological Sciences, Ahmadu Bello University Zaria, Nigeria and at the National Institute for Pharmaceutical Research and Development, Idu-Abuja, Nigeria where the voucher specimens have been deposited in the Herbaria (Table 1).

\section{Statistical analysis}

Performance index adopted for this study is based on the studies of the medicinal plants of Baka Pygmies [12]. It is an analytical tool for assessing the effectiveness of medicinal plants. For analyzing the data, "Specific flora" is defined as the list of plants used for treating a specific ailment, symptom and physiological manifestations. The "global flora" is defined as the total list of plants recorded in use for all types of ailments among a specific group, which are the three major tribes of Niger State. The relationship between the "specific flora" and the "global flora" can be inferred as follows; if the use of a specific plant for a specific ailment is randomly selected, the proportion (P1) of the number of its citations for the specific ailment $(\mathrm{C} 1)$ to the total number of its citations $(\mathrm{C} 2)$ in the group would be similar to the proportion $(\mathrm{P} 2)$ of the citations $(\mathrm{C} 3)$ of the specific ailment in the group to the total number of citations $(\mathrm{C} 4)$ of all ailments in the group. To illustrate the selectivity of a plant for a specific ailment, comparison is made here between the expected and observed values of the proportion of citation of a plant for a specific 
disease. The difference (D) between the two proportions is then used to define a performance index (Ip), which ranges from 0 to 3 according to the following arbitrary scale.

If $\mathrm{P} 1 \mathrm{P} 2<0, I p=0$ : the plants concerned are rejected, not significant If $0<\mathrm{P} 1 \mathrm{P} 21 / 3, I p=1$ : average performance;

If $1 / 3<\mathrm{P} 1 \mathrm{P} 2{ }_{3}{ }_{3}$, I $p=2$ : high performance;

If P1 P $2>{ }_{3}^{2}, I p=3$ : very high performance as by described the method of $\operatorname{Betti}(2004)[12]$.

To illustrate this, an example is given for the performance index of a plant, Vernonia amygdalina used for the treatment of cough (specific ailment). The group is all respiratory ailments.

$\mathrm{C}_{1}=$ number of citations of Vernonia amygdalina for treating cough $=1$;

$\mathrm{C}_{2}=$ number of citations of Vernonia amygdalina in the global list (all respiratory ailments) $=4$

$\mathrm{C}_{3}=$ total number of citations of the cough $=89$

$\mathrm{C}_{4}=$ total number of citations for all respiratory ailments $=409$.

P1 (observed) and P2 (theoretical) are calculated as follows:

$\mathrm{P} 1={ }^{\mathrm{C} 1} / \mathrm{C}_{2}=1 / 4=0.25$

$\mathrm{P} 2={ }^{\mathrm{C} 3} / \mathrm{C}_{4}={ }^{89} / 409=0.22$

$\mathrm{D}=\mathrm{P} 1 \mathrm{P} 2=0.250 .22=0.03$

P1 P2 $=0.03<1 / \frac{1}{3}$, therefore, $I p=1$, which denotes an average performance.

The proportions used are calculated from the ratios of number of citations. The number of citations for each plant species used for a specific ailment is recorded.

\section{Results}

Three visits were undertaken during the study involving 13 Local Government Areas (LGA) in Niger State and Patigi Local Government in Kwara State due to the cultural similarity and language affinity. The first trip took place during the period of November to December 2004 and seven Local Government Areas were randomly selected for coverage in the southern zone due to high population density in this zone. The Local Government Areas visited in this zone with the number of respondents indicated in brackets comprise of Agaie (1), Bida (4), Gbako (5), Katcha (2), Lavun (10), Mokwa (1) and Patigi (2). The second visit was undertaken in the second week of January 2005 and it covered three Local Government Areas comprising of Bosso (4), Minna (4) and Paikoro (3) from the eastern geographical zone. The third visit took place from $28^{\text {th }}$ January to $5^{\text {th }}$ March, 2005 and it covered 
three Local Government Areas of Kontagora (3), Mariga (9) and Rijau (4) from the northern geographical zone. During the three visits altogether fifty-two herbal informants were surveyed. The interviews were done in both urban and rural areas of Niger State.

The interviewers principally made use of the members of the existing association called Nigerian Association of Traditional Medical Practitioners and non-members in some cases in the markets of Bida, Minna and Kontagora. A total of 95 plant species belonging to 48 families were identified from 86 herbal recipes of Niger State (Table 1). The performance indices for all the identified plants from the study area are recorded (Table 2). Some prescriptions are made of two or more plant species (Table 1). Most recipes involved admixture of plants; one or more plants being considered as major ingredients and others as additives (Table 1). In all the recipes, water was used for the extraction either by infusion (cold maceration) or decoction (boiling). Dosages were by teaspoonful $(5 \mathrm{ml})$, tablespoonful $(10 \mathrm{ml})$, teacupful $(200 \mathrm{ml})$ and table cupful $(400 \mathrm{ml})$ which is often to be taken twice or thrice daily from one week to six months. The information on the therapeutic uses of medicinal plants for respiratory diseases is classified according to the WHO guidelines employed by Betti [12]. Traditional medical practitioners and herbal traders mostly provided the information because they possess good knowledge of medicinal plants. They provided useful, reliable and firsthand information on the common plants that are used in the treatment of anti-TB and other respiratory diseases. There was no problem in communication because some members of the study team are indigenes of Niger State, and could speak and understand the local languages fluently.

A total of 409 citations of medicinal plants were recorded for 12 respiratory diseases as summarized in Table 3. In terms of plant families Euphorbiaceae had the highest number of plants prescribed to be 7 species, followed by Loranthaceae having 6 species; other families that more frequently represented are Caesalpinaceae (5), Papilionaceae (5), Poaceae (4), Mimosaceae (4), Scrophulariaceae (4), Anacardiaceae (3), Combretaceae (3), Liliaceae (3) and Solanaceae (3); with figure in bracket representing the number of species cited per family. 


\begin{tabular}{|c|c|c|c|c|c|}
\hline$\underset{\text { number }^{a}}{\text { Herbarium }}$ & $\begin{array}{l}\text { Families/Botanical Names of } \\
\text { Plant Species }\end{array}$ & $\begin{array}{l}\text { Common/English } \\
\text { names }\end{array}$ & Local Nigerian Names ${ }^{\mathrm{b}}$ & $\begin{array}{l}\text { Part Used/ } \\
\text { Nature of } \\
\text { ingredient }^{\mathrm{c}}\end{array}$ & Tribes $^{d}$ \\
\hline 184 & Anacardium occidentale L. & Cashew & $\begin{array}{l}\text { Kashiwu/Ekonasara(N), Kashew(H), } \\
\text { Kaju (Y) } \\
\text { Kausu(I) }\end{array}$ & sb,l;ma & $2 \mathrm{~N}$ \\
\hline (5744) & $\begin{array}{l}\text { Lannea kerstingii Engl. \& } \\
\text { K.Krause }\end{array}$ & Wodier wood & $\begin{array}{l}\text { Yinci (N), Ekika(Y), Vyari (G), Faru } \\
(\mathrm{H})\end{array}$ & sb;ma & $2 \mathrm{~N}$ \\
\hline \multirow[t]{2}{*}{2384} & Spondias mombin $\mathrm{L}$. & Hog plum & $\begin{array}{l}\text { Jinjereci (N), Gbabanri (G), Tsadar } \\
\text { masar9H), Akkika(Y), } \\
\text { Ishikala/ogaga/Jinkara(I) }\end{array}$ & sb;ma & $1 \mathrm{~N}$ \\
\hline & Annonaceae & & & & \\
\hline (5737) & Annona senegalensis Perr. & Soursop & $\begin{array}{l}\text { Nungbereci (N), Gwandardaji (H), } \\
\text { Laabo (Y), Uhuru-ocha (I), } \\
\text { Kocekoceyi (G) }\end{array}$ & $r ; m a$ & $\begin{array}{l}1 \mathrm{~N}, \\
3 \mathrm{H}\end{array}$ \\
\hline 7 & $\begin{array}{l}\text { Xylopia aethiopica (Dunal) } \\
\text { A.Rich } \\
\text { Araceae }\end{array}$ & $\begin{array}{l}\text { African pepper, } \\
\text { Guinea pepper, } \\
\text { Ethiopia pepper }\end{array}$ & $\begin{array}{l}\text { Tsunfyania (N), Kimba (H), Erunje } \\
\text { (Y), } \\
\text { Uda (I), Sumo (G) }\end{array}$ & $\mathrm{f} ; \mathrm{ma}$ & $2 \mathrm{~N}$ \\
\hline 900283 & $\begin{array}{l}\text { Anchomanes difformis (BL) } \\
\text { Engl. }\end{array}$ & $\begin{array}{l}\text { The forest } \\
\text { Anchomanes }\end{array}$ & $\begin{array}{l}\text { Hantsar-gada }(\mathrm{H}) \text {, Okukuigo }(\mathrm{Y}) \text {, Oje } \\
\text { (I) }\end{array}$ & $\mathrm{r} ; \mathrm{ma}$ & $1 H^{1 G}$ \\
\hline 1977 & $\begin{array}{l}\text { Pistia stratiotes L. } \\
\text { Araliaceae }\end{array}$ & Water lettuce & $\begin{array}{l}\text { Sanbogi }(\mathrm{N}) \text {, Kainuwa }(\mathrm{H}) \text {, Ejuoro } \\
(\mathrm{Y})\end{array}$ & wp;ad & $1 \mathrm{~N}$ \\
\hline \multirow[t]{2}{*}{193} & $\begin{array}{l}\text { Cussonia arborea Hochst ex. } \\
\text { A.Rich } \\
\text { (Syn:.Cussonia barteri) }\end{array}$ & $\begin{array}{l}\text { Barters cussunia, } \\
\text { Cork - bark tree }\end{array}$ & $\begin{array}{l}\text { Kpawu/Efokoci }(\mathrm{N}) \text {, Takandargiwa } \\
(\mathrm{H}) \text {, Shigo }(\mathrm{Y})\end{array}$ & $\mathrm{sb} ; \mathrm{m}$ & $1 \mathrm{~N}$ \\
\hline & Asclepiaceae & & & & \\
\hline 369 & Calotropis procera (Ait) Ait.f. & $\begin{array}{l}\text { Sodom apple, } \\
\text { Swallow wart }\end{array}$ & $\begin{array}{l}\text { Epuko }(\mathrm{N}) \text {, Tumfafiya }(\mathrm{H}) \text {, } \\
\text { Bomubomu (Y), } \\
\text { Bombongo }(\mathrm{G})\end{array}$ & $\mathrm{r}, \mathrm{f} ; \mathrm{ma}$ & $3 \mathrm{~N}$ \\
\hline \multirow[t]{2}{*}{675} & $\begin{array}{l}\text { Asteraceae } \\
\text { Vernonia amygdalina Del }\end{array}$ & Bitter leaf & $\begin{array}{l}\text { Tsula }(N) \text {, Susu }(G) \text {, Shiwaka }(H) \text {, } \\
\text { Ewuro (Y), } \\
\text { Onugbu/Olubo (I) }\end{array}$ & I;ma & $2 \mathrm{~N}$ \\
\hline & Bignoniaceae & & & & \\
\hline 2881 & $\begin{array}{l}\text { Newboldia laevis (P.Beauv) } \\
\text { Seaman ex. } \\
\text { Bunean }\end{array}$ & $\begin{array}{l}\text { Tree of life, fertility } \\
\text { tree }\end{array}$ & $\begin{array}{l}\text { Dinberechiamile (N), Shigbelogoye } \\
(\mathrm{G}) \text {, Aduruku }(\mathrm{H}) \text {, Akoro }(\mathrm{Y}) \text {, Ogirisi } \\
(\mathrm{I})\end{array}$ & sb;ma & $1 \mathrm{~N}$ \\
\hline \multirow[t]{2}{*}{1381} & $\begin{array}{l}\text { Stereospermum kunthianum } \\
\text { Cham }\end{array}$ & $\begin{array}{l}\text { Kunth's } \\
\text { stereospermum }\end{array}$ & $\begin{array}{l}\text { Dagbapanboci }(\mathrm{N}) \text {, Kushishigban } \\
(\mathrm{G}) \text {, Jiri/Sansami }(\mathrm{H}) \text {, } \\
\text { Ayada/Erumeye }(\mathrm{Y})\end{array}$ & sb;ma & $2 \mathrm{~N}, 1 \mathrm{G}, 1 \mathrm{H}$ \\
\hline & Caesalpiniaceae & & & & \\
\hline 6907 & $\begin{array}{l}\text { Daniellia oliveri (Rolfe) Hutch \& } \\
\text { Dalz. }\end{array}$ & Ilorin balsam & $\begin{array}{l}\text { Danci (N), Dayi (G), Kadaura/Maje } \\
(\mathrm{H}) \text {, } \\
\text { lya (Y), Ozabwa (I) }\end{array}$ & m;ma & $2 \mathrm{~N}$ \\
\hline 900676 & $\begin{array}{l}\text { Detarium microcarpum Guill \& } \\
\text { Perr. }\end{array}$ & & $\begin{array}{l}\text { Gungoroci (N), Gongoyi }(G) \text {, Taura } \\
(\mathrm{H}) \text {, } \\
\text { Ofo (I), Sedun/Ogbogbo }(\mathrm{Y})\end{array}$ & $\mathrm{sb} ; \mathrm{ma}$ & $3 \mathrm{~N}, \quad 1 \mathrm{G}, 1 \mathrm{H}$ \\
\hline (5745) & $\begin{array}{l}\text { Piliostigma thonningii (Schum) } \\
\text { Milne-Red Head }\end{array}$ & $\begin{array}{l}\text { Thonning's } \\
\text { piliostigma }\end{array}$ & $\begin{array}{l}\text { Bafin }(\mathrm{N}) \text {, Kalgo }(\mathrm{H}) \text {, Abafe }(\mathrm{Y}) \text {, } \\
\text { Okpoatu (I) Tawegi }(\mathrm{G})\end{array}$ & $\operatorname{tg}, r ; m a$ & $\begin{array}{l}1 \mathrm{~N}, \\
3 \mathrm{H}\end{array}$ \\
\hline 1370 & $\begin{array}{l}\text { Senna obtusifolia (L) Irwin. \& } \\
\text { Barney } \\
\text { (Syn: Cassia tora L). }\end{array}$ & Foetid cassia & $\begin{array}{l}\text { Gaya-eba }(N) \text {, Tafasa }(H), \\
\text { Jelenubenu }(Y), \\
\text { Gaya }(G)\end{array}$ & $\operatorname{tg} ; m a$ & $\begin{array}{l}2 \mathrm{~N}, \\
1 \mathrm{H}\end{array}$ \\
\hline
\end{tabular}


Abdullahi, M. et al

jopat 1220071 - 21

\begin{tabular}{|c|c|c|c|c|c|c|}
\hline 900265 & Tamarindus indica L. & $\begin{array}{l}\text { Tamarina tree, } \\
\text { Indian tamarind }\end{array}$ & $\begin{array}{l}\text { Daraci (N), Dandayi (G), Tsamiya } \\
\text { (H), } \\
\text { Aagbon (Y), Icheku - oyibo (I) }\end{array}$ & riad & $1 \mathrm{G}$ & \\
\hline & Capparidaceae & & & & & \\
\hline $\begin{array}{l}(5750) \\
6967\end{array}$ & $\begin{array}{l}\text { Capparis brassii DC } \\
\text { Crateva adansonii DC } \\
\text { (Syn: Crateva religiosa Forst f. } \\
\text { Aedres) } \\
\text { Caricaceae }\end{array}$ & & $\begin{array}{l}\text { Ekanchi-wuriagi (N) } \\
\text { Kulanci, (G) Unguduudu/Kalu/Gudai } \\
\text { (H), Oto/Eegun oru (Y) }\end{array}$ & $\begin{array}{l}\mathrm{rb}, \mathrm{ma} \\
\mathrm{r}, \mathrm{ma}\end{array}$ & $\begin{array}{l}1 \mathrm{~N} \\
2 \mathrm{~N}\end{array}$ & \\
\hline 72 & Carica papaya L & Pawpaw & $\begin{array}{l}\text { Konkeni }(\mathrm{N}) \text {, Gwanda }(\mathrm{H}) \text {, } \\
\text { Ojo/Okwwere }(\mathrm{I}) \text {, } \\
\text { lbepe }(\mathrm{Y})\end{array}$ & l;ma & $4 \mathrm{~N}$ & \\
\hline & Cleaomaceae & & & & & \\
\hline 576 & Cleome viscose $\mathrm{L}$ & $\begin{array}{l}\text { Chicken weed, } \\
\text { Consumption weed }\end{array}$ & $\begin{array}{l}\text { Emigi (N), Epia/Ekuya (Y), } \\
\text { llulokwonwa (I) }\end{array}$ & wp; ma & $4 \mathrm{~N}$ & \\
\hline (5736) & Combretum spp. & & Kukunci (N), & $\mathrm{rb}, \mathrm{sb} ; \mathrm{ma}$ & $\begin{array}{l}3 \mathrm{~N}, \\
1 \mathrm{H}\end{array}$ & $3 G$ \\
\hline (5735) & $\begin{array}{l}\text { Terminalia avicennioides Guill } \\
\text { \& Perr. (Syn: Terminalila } \\
\text { schimperiana Hochst, } \\
\text { Terminalia glaucescens Planch } \\
\text { Ex Bth) }\end{array}$ & & $\begin{array}{l}\text { Kpace (N), Kpayi (G), Baushe (H), } \\
\text { Igiodan (Y), Edo (I) }\end{array}$ & $m, r b, f ; m a$ & $\begin{array}{l}10 \mathrm{~N}, \\
1 \mathrm{H}\end{array}$ & $1 \mathrm{G}$ \\
\hline 167 & $\begin{array}{l}\text { Anogeissus leiocarpus (DC) } \\
\text { Guill \& Perr. } \\
\text { Convovulaceae }\end{array}$ & Axle-wood tree & $\begin{array}{l}\text { Shici (N), Suasuanveyi (G), Marike } \\
(\mathrm{H}) \text {, } \\
\text { Ayin (Y), Atara (I) }\end{array}$ & sb;ma & $\begin{array}{l}3 \mathrm{~N}, \\
2 \mathrm{H}\end{array}$ & $1 \mathrm{G}$ \\
\hline 158 & Ipomoea aquatica Forsk & $\begin{array}{l}\text { Water spinach, } \\
\text { Swamp morning } \\
\text { glory }\end{array}$ & $\begin{array}{l}\text { Daman (N), Dama (G), } \\
\text { Awosawarool } \\
\text { Furengadu (H), Odunkun-odo (Y) }\end{array}$ & wp;ma & $1 \mathrm{~N}$ & \\
\hline & Cucurbitaceae & & & & & \\
\hline 525 & $\begin{array}{l}\text { Luffa cylindrica (L) M. J. Roem } \\
\text { Cyperaceae }\end{array}$ & Sponge plant & $\begin{array}{l}\text { Kankani }(\mathrm{N}) \text {, Karinkan }(\mathrm{Y}) \text {, Kakanyi } \\
(\mathrm{G})\end{array}$ & ssh;ma & $1 \mathrm{~N}$ & \\
\hline 900714 & $\begin{array}{l}\text { Fuirena umbellata Rotb. } \\
\text { Dioscoraceae }\end{array}$ & & Gozanko (N), Yagoyi (G) & ap;ma & & $1 \mathrm{G}$ \\
\hline 901467 & Dioscorea abyssinica $L$. & & Eciko-kana (N) & ap;ma & $1 \mathrm{~N}$ & \\
\hline & Euphorbiaceae & & & & & \\
\hline 1665 & $\begin{array}{l}\text { Alchornea cordifolia Schum \& } \\
\text { Thonn Muell.Arg }\end{array}$ & Christmas bush & $\begin{array}{l}\text { Ububo (I), Bambami (H), Dzuzanci } \\
\text { (N), } \\
\text { Ipa/Esinyin (Y) }\end{array}$ & ap;ma & $\begin{array}{l}1 \mathrm{~N}, \\
1 \mathrm{H}\end{array}$ & \\
\hline 937 & Bridelia ferruginea Bth. & & Kirni/Kishi (H), Iraodan (Y), Ola (I) & sb,r,ma & $\begin{array}{l}1 \mathrm{~N}, \\
1 \mathrm{H}\end{array}$ & \\
\hline 579 & $\begin{array}{l}\text { Chlorophora senegalensis } \\
\text { (Lam) A.Juss.ex Spreng }\end{array}$ & & $\begin{array}{l}\text { Damagi (N,G,H,) Damaigi/Bauren } \\
\text { Kiyashi(H) }\end{array}$ & ap,ma & $\begin{array}{l}{ }_{1}^{N}, \\
1 H\end{array}$ & \\
\hline 583 & Euphorbia hirta L. & Asthma herb & $\begin{array}{l}\text { Odane inenemili (I), Kinkerechintara } \\
(\mathrm{N}) \text {, } \\
\text { Nonknkurciya }(\mathrm{H}) \text {, Emile/Egele }(\mathrm{Y}) \text {, }\end{array}$ & wp;ma & $\begin{array}{l}1 \mathrm{~N}, \\
1 \mathrm{H}\end{array}$ & \\
\hline (5741) & Hymenocardia acida Tul. & & Lupka (N), Janyaro (H) & r,ma & $3 \mathrm{~N}$, & $1 \mathrm{G}, 1 \mathrm{H}$ \\
\hline 1911 & Jatropha curcas $\mathrm{L}$ & Pignut, Fignut & $\begin{array}{l}\text { Kasha'a }(\mathrm{N}) \text {, Kpotobiyi }(\mathrm{G}) \\
\text { Halallamaiai/Binidazugu }(\mathrm{H}) \text {, } \\
\text { Lapalana/Botuje }(\mathrm{Y})\end{array}$ & $\mathrm{r}, \mathrm{l} ; \mathrm{ma}$ & $2 \mathrm{~N}$, & \\
\hline 932 & Ricinus communis $\mathrm{L}$ & Castor oil plant & Kpanafinikogulu (N), Dankwasare & sd,l;ma & $2 \mathrm{~N}$ & \\
\hline
\end{tabular}


Abdullahi, M. et al

jopat 1220071 - 21

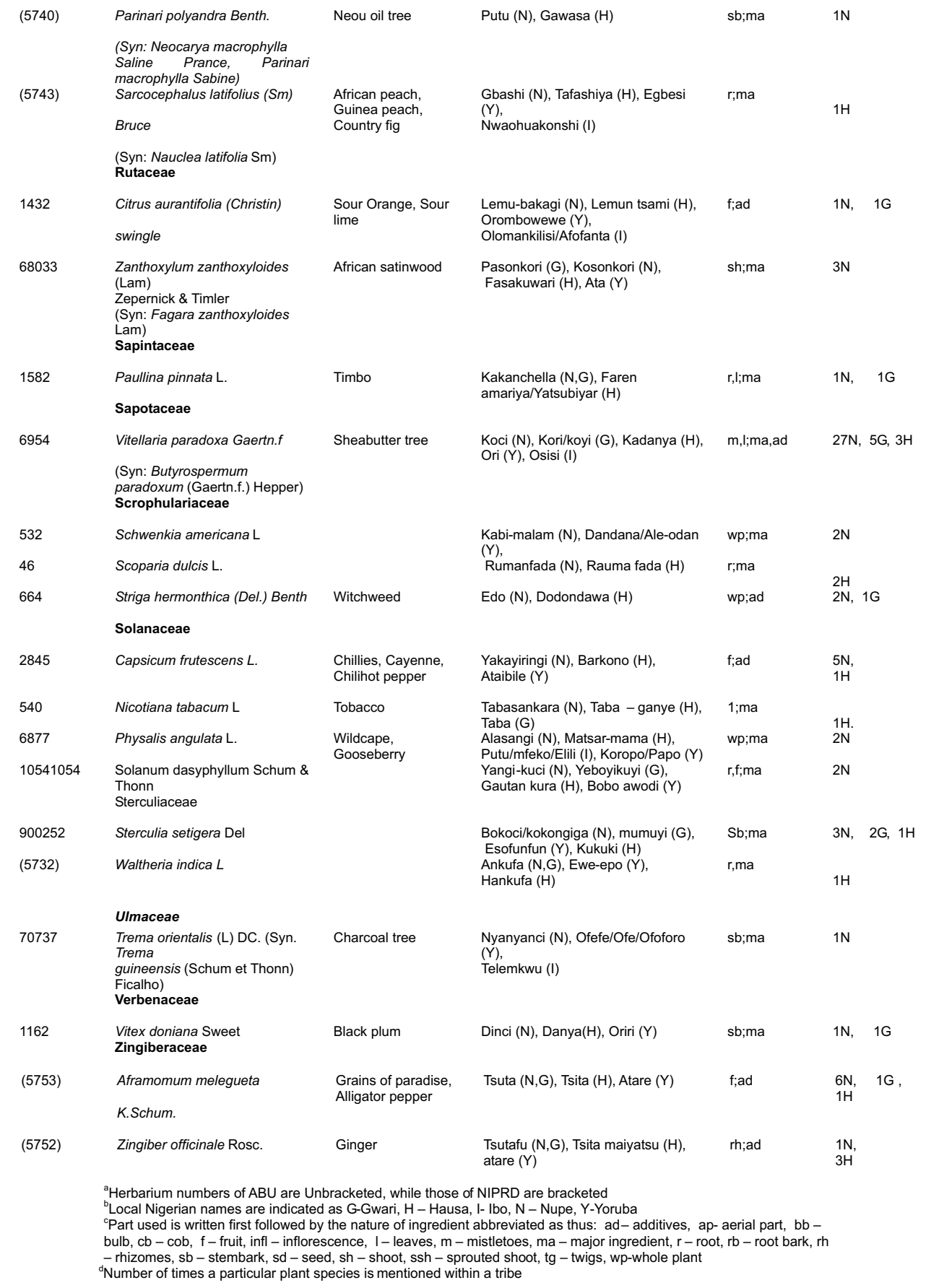


Table 2: Index of performance of Plants with Anti-tuberculosis and activities on other respiratory diseases from Niger State, Nigeria

Plant Species

Anacardium occidentale L.

Lannea bateri Kokwara \& Guilet

Spondias mombin L.

Annona senegalensis Perr.

Xylopia aethiopica (Dunal) A.Rich

Anchomanes difformis (BL) Engl.

Pistia stratiotes L.

Cussonia arborea Hochst ex. A.Rich

Syn.Cussonia barteri

Calotropis procrea (Ait) Ait.f.

Newboldia laevis (P.Beauv) Seaman ex. Bunean

Stereospermum kunthianum Cham

Daniellia oliveri (Rolfe) Hutch \& Dalz.

Detarium microcarpum Guill \& Perr.

Piliostigma thonningii (Schum) Milne-Red Head

Senna obtusifolia (L) Irwin. \& Barney Syn.

Cassia tora $L$.

Tamarindus indica $\mathrm{L}$.

Crateva adansonii DC Syn.Crateva religiosa

Forst f. Aedres

Carica papaya $\mathrm{L}$

Cleome viscose $\mathrm{L}$

Combretum spp

Terminalia avicennioides Guill \& Perr

Anogeissus leiocarpus (DC) Guill \& Perr

Vernonia amygdalina Del

Ipomoea aquatica Forsk

Luffa cylindrica (L) M. J. Roem

Fuirena umbellate Rotb.

Dioscorea abyssinica $L$

$\begin{array}{lllllllllllll}\text { As } & \mathbf{B r} & \mathbf{C a} & \mathbf{C d} & \mathbf{C o} & \mathbf{C p} & \mathbf{E x} & \mathbf{F e} & \mathbf{H e} & \mathbf{S t} & \mathbf{T b} & \mathbf{W c} & \text { Total } \\ 0 & 0 & 0 & 0 & 0 & 0 & 0 & 0 & 0 & 0 & 2 & 0 & 2 \\ 1 & 0 & 0 & 0 & 0 & 0 & 0 & 0 & 0 & 0 & 1 & 0 & 5 \\ 1 & 0 & 0 & 0 & 0 & 0 & 0 & 0 & 0 & 0 & 1 & 0 & 2 \\ 0 & 0 & 0 & 0 & 0 & 0 & 2 & 0 & 0 & 0 & 2 & 0 & 5 \\ 0 & 0 & 0 & 0 & 0 & 0 & 0 & 0 & 0 & 0 & 2 & 0 & 2 \\ 0 & 0 & 0 & 0 & 0 & 0 & 0 & 0 & 0 & 0 & 2 & 0 & 2 \\ 0 & 0 & 0 & 0 & 0 & 0 & 0 & 0 & 0 & 0 & 2 & 0 & 1 \\ 0 & 0 & 0 & 0 & 0 & 0 & 0 & 0 & 2 & 0 & 0 & 2 & 2 \\ 1 & 0 & 0 & 0 & 0 & 0 & 0 & 0 & 0 & 0 & 1 & 0 & 5 \\ 0 & 0 & 0 & 0 & 0 & 0 & 0 & 0 & 3 & 0 & 0 & 0 & 1 \\ 0 & 0 & 0 & 0 & 0 & 0 & 0 & 0 & 0 & 0 & 2 & 0 & 4 \\ 0 & 3 & 0 & 0 & 0 & 0 & 0 & 0 & 0 & 0 & 0 & 0 & 2\end{array}$

Alchornea cordifolia Schum \& Thonn Muell.Arg

Bridelia ferruginea Bth.

Chrozophora senegalensis (Lam) A.Juss.ex

Spreng

Euphorbia hirta L.

Hymenocardia acida Tul.

$\begin{array}{lllllllllllll}1 & 0 & 0 & 0 & 1 & 0 & 0 & 0 & 1 & 0 & 0 & 0 & 13 \\ 0 & 0 & 0 & 0 & 0 & 1 & 0 & 0 & 1 & 1 & 0 & 0 & 10\end{array}$

Jatropha curcas L

Ricinus communis $\mathrm{L}$

Pennisetum typhoides Burm.f. Stapf. \& Hubbard

Andropogan gayanus Kunth

$\begin{array}{lllllllllllll}0 & 3 & 0 & 0 & 0 & 0 & 0 & 0 & 0 & 0 & 0 & 0 & 1 \\ 2 & 0 & 0 & 0 & 0 & 0 & 0 & 0 & 0 & 0 & 1 & 0 & 2\end{array}$

$\begin{array}{lllllllllllll}0 & 0 & 0 & 0 & 1 & 0 & 0 & 0 & 0 & 0 & 1 & 0 & 4\end{array}$

$\begin{array}{lllllllllllll}1 & 0 & 0 & 0 & 1 & 1 & 0 & 0 & 0 & 0 & 0 & 0 & 8 \\ 0 & 1 & 0 & 0 & 1 & 0 & 0 & 0 & 0 & 0 & 1 & 0 & 9\end{array}$

$\begin{array}{lllllllllllll}0 & 1 & 0 & 0 & 0 & 0 & 0 & 0 & 1 & 0 & 1 & 0 & 14 \\ 0 & 0 & 0 & 0 & 0 & 0 & 0 & 0 & 0 & 0 & 2 & 0 & 6\end{array}$

$\begin{array}{lllllllllllll}1 & 0 & 0 & 0 & 1 & 0 & 0 & 0 & 0 & 0 & 1 & 0 & 4 \\ 1 & 0 & 0 & 0 & 1 & 0 & 1 & 0 & 0 & 0 & 0 & 0 & 3\end{array}$

$\begin{array}{lllllllllllll}0 & 0 & 0 & 0 & 0 & 0 & 0 & 0 & 3 & 0 & 0 & 0 & 1 \\ 0 & 0 & 0 & 0 & 0 & 0 & 0 & 0 & 3 & 0 & 0 & 0 & 1\end{array}$

$\begin{array}{lllllllllllll}0 & 0 & 0 & 0 & 0 & 0 & 0 & 0 & 0 & 0 & 2 & 0 & 1\end{array}$

$\begin{array}{lllllllllllll}0 & 0 & 0 & 0 & 0 & 0 & 0 & 0 & 0 & 0 & 2 & 0 & 2 \\ 0 & 0 & 0 & 0 & 0 & 0 & 0 & 0 & 0 & 0 & 0 & 3 & 2 \\ 0 & 0 & 0 & 0 & 0 & 0 & 0 & 0 & 0 & 0 & 0 & 3 & 2\end{array}$

Imperata cylinderica (Anderes) Hubbard

Zea mays L.

Garcinia kola Heckel

Allium ascalonicum $L$

Allium sativum $\mathrm{L}$

$\begin{array}{lllllllllllll}1 & 0 & 0 & 0 & 1 & 0 & 0 & 0 & 0 & 0 & 0 & 0 & 6 \\ 0 & 0 & 0 & 0 & 0 & 0 & 0 & 0 & 1 & 0 & 1 & 0 & 7 \\ 0 & 0 & 0 & 0 & 0 & 0 & 0 & 0 & 0 & 0 & 2 & 0 & 2 \\ 0 & 0 & 0 & 0 & 0 & 0 & 0 & 0 & 0 & 0 & 2 & 0 & 2 \\ 0 & 0 & 0 & 0 & 1 & 0 & 0 & 0 & 0 & 0 & 1 & 0 & 2 \\ 0 & 0 & 0 & 0 & 1 & 0 & 0 & 0 & 2 & 0 & 0 & 0 & 2 \\ 1 & 0 & 0 & 0 & 1 & 0 & 0 & 0 & 1 & 0 & 0 & 0 & 3 \\ 0 & 0 & 0 & 1 & 1 & 0 & 0 & 1 & 0 & 0 & 0 & 0 & 3 \\ 0 & 0 & 0 & 0 & 1 & 0 & 0 & 0 & 0 & 0 & 1 & 0 & 4 \\ 1 & 0 & 0 & 0 & 1 & 0 & 0 & 0 & 1 & 0 & 0 & 0 & 11 \\ 1 & 0 & 0 & 0 & 1 & 0 & 0 & 0 & 0 & 0 & 1 & 0 & 12\end{array}$


Ocimum gratissimum L.

Anthocleista vogelii Planch

\section{Ammania auriculata Wild}

Lawsonia inermis (L) Keay

Hibiscuss asper Hook.

Azadirachta indica A. Jus

Khaya senegalensis (Desr.) A. Juss

Acacia nilotica (L) Wild \& Del

Entada africana Guill \& Ferr.

Parkia biglobosa Aubrevielle

Prosopis africana (Guill \& Perr) Taub

Milicia excelsa (Welw.) CCBerg Syn.Chlorophora excelsa (Welw.)Bth

Moringa oleifera L

Psidium guajava L

Boerhavia diffusa L

Nymphaea lotus L

Olax subscorpiodea Oliv.

Argemone mexicana L

Elaeis guineensis Jacq

Borassus flabelifer var. aethiophian Warb.

Abrus precatorius $\mathrm{L}$

Calopogonium mucunoides Desr

Erythrina senegalensis DC

Mucuna poggei L.

Pterocarpus erinaceus Poir

Piper guineense Schum \& Thonn

Securidaca longepedunculata Frer.

Capparis brassii Dc

Gardenia ternifolia Schum \& Thonn

Neocarya macrophylla Saline Prance

Syn. Parinari macrophylla Sabine

Sarcocephalus latifolius (Sm) Bruce

Syn. Nauclea latifolia $\mathrm{Sm}$

Citrus aurantifolia (Christin) swingle

Zanthoxylum zanthoxyloides (Lam) Zep.\& T. Syn. Fagara zanthoxyloides Lam

zanthoxyloides Lam

Vaullina pinnata $L$.

Syn B. paradoxum (Gaertn.f.) Hepper

Schwenkia Americana L

Scoparia dulcis $\mathrm{L}$.

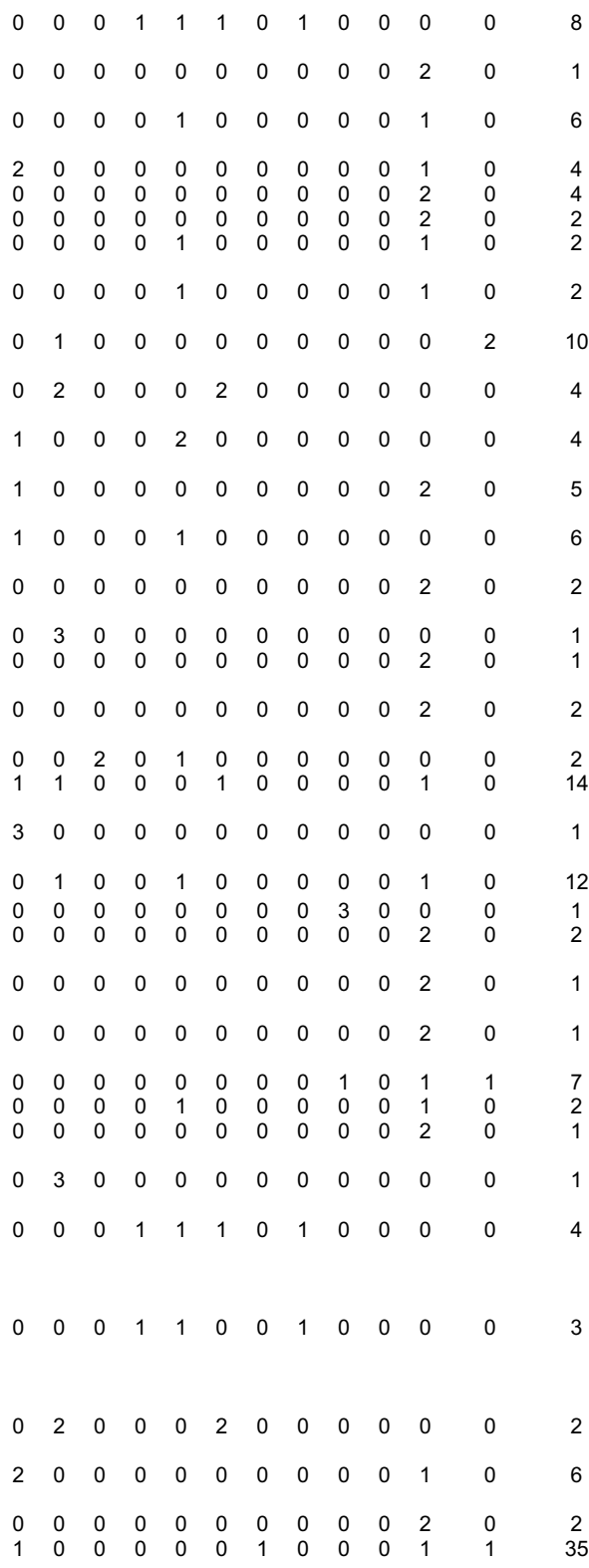

$\begin{array}{lllllllllllll}0 & 0 & 0 & 0 & 0 & 0 & 0 & 0 & 0 & 0 & 2 & 0 & 2 \\ 0 & 0 & 0 & 0 & 1 & 1 & 0 & 0 & 1 & 1 & 0 & 0 & 8\end{array}$ 
Abdullahi, M. et al

jopat $1220071-21$

Solanum dasyphyllum Schum \& Thonn

Striga hermonthica (Del.) Benth

Capsicum frutescens $L$.

Nicotiana tabacum L

Physalis angulata $\mathrm{L}$.

Sterculia setigera De

Waltheria indica $L$

$\begin{array}{ccccccccccccc}1 & 0 & 0 & 0 & 0 & 0 & 0 & 0 & 0 & 0 & 1 & 0 & 3 \\ 1 & 0 & 0 & 0 & 1 & 0 & 0 & 0 & 1 & 0 & 0 & 0 & 9 \\ 0 & 0 & 0 & 0 & 1 & 0 & 0 & 0 & 1 & 0 & 0 & 0 & 9 \\ 0 & 3 & 0 & 0 & 0 & 0 & 0 & 0 & 0 & 0 & 0 & 0 & 1 \\ 0 & 0 & 0 & 0 & 0 & 0 & 0 & 0 & 0 & 0 & 2 & 0 & 2 \\ 1 & 1 & 0 & 0 & 1 & 0 & 0 & 0 & 1 & 0 & 0 & 0 & 15 \\ 0 & 0 & 0 & 0 & 0 & 0 & 0 & 0 & 0 & 0 & 2 & 0 & 1\end{array}$

Trema orientalis (L) Dc. Syn. Trema guineensis (Schum et Thonn) Ficalho

$\begin{array}{lllllllllllll}0 & 0 & 0 & 0 & 1 & 0 & 0 & 2 & 0 & 0 & 0 & 0 & 2\end{array}$

Vitex doniana Sweet

Aframomum melegueta K.Schum.

$\begin{array}{lllllllllllll}0 & 0 & 0 & 0 & 0 & 0 & 0 & 0 & 0 & 0 & 2 & 0 & 2 \\ 0 & 1 & 0 & 1 & 0 & 0 & 1 & 1 & 0 & 0 & 0 & 0 & 10\end{array}$

Zingiber officinale Rosc

Total number of citation

$\begin{array}{llllllllllllr}0 & 0 & 0 & 1 & 1 & 0 & 0 & 1 & 0 & 0 & 0 & 0 & 8 \\ 51 & 21 & 1 & 6 & 89 & 13 & 5 & 7 & 32 & 4 & 166 & 14 & 409\end{array}$

$\begin{array}{lllllllllllll}51 & 21 & 1 & 6 & 89 & 13 & 5 & 7 & 32 & 4 & 166 & 14 & 409\end{array}$

Aliments: $\mathrm{As}=$ asthma; $\mathrm{Br}=$ bronchitis; $\mathrm{Ca}=$ catarrh; $\mathrm{Cd}=$ colds; $\mathrm{Co}=$ cough; $\mathrm{Cp}=$ Chest pains; $\mathrm{Ex}=$ expectorant; $\mathrm{Fe}=$ fever; $\mathrm{He}=$ hemoptysis; $\mathrm{St}=$ sore throat; $\mathrm{Tb}=$ tuberculosis; $\mathrm{Wc}=$ whooping cough.

$I p=$ denotes the performance index, which is the difference (D) between expected and observed values of the proportion of citation of a plant for a specific disease; it ranges from 0 to 3. P1 (observed) and P2 ( expected) are calculated as follows: $\mathrm{P} 1={ }_{11} / \mathrm{C2}_{2}, \mathrm{P} 2=\mathrm{C}_{3} / \mathrm{C} 4 ; \mathrm{D}=\mathrm{P} 1-\mathrm{P} 2$. Where $\mathrm{C}_{1}=$ number of citations of plant for treating specific ailment, $\mathrm{C}_{2}=$ number of citations of plant in the global list (all ailments),$C_{3}=$ total number of citations of the specifi $c$ ailment, $C_{4}=$ total number of citations for all ailments. Ip $=0$ : the plants concerned are rejected, not significant, Ip= 1: average performance, number of citations for all ailments. Ip $=0$ : the plants conce
Ip $=2$ : high performance, $I p=3$ : very high performance.

Table 3: Citations of respiratory diseases in Niger State, Nigeria

\begin{tabular}{|l|r|}
\hline Respiratory Diseases & Number of Citations \\
\hline Asthma & 51 \\
Bronchitis & 21 \\
Catarrh & 1 \\
Cold & 6 \\
Cough & 89 \\
Chest pain & 13 \\
Expectorant & 5 \\
Fever & 7 \\
Hemoptysis & 32 \\
Sore throat & 4 \\
Tuberculosis & 166 \\
Whooping Cough & 14 \\
Total number of citations & 409 \\
\hline
\end{tabular}




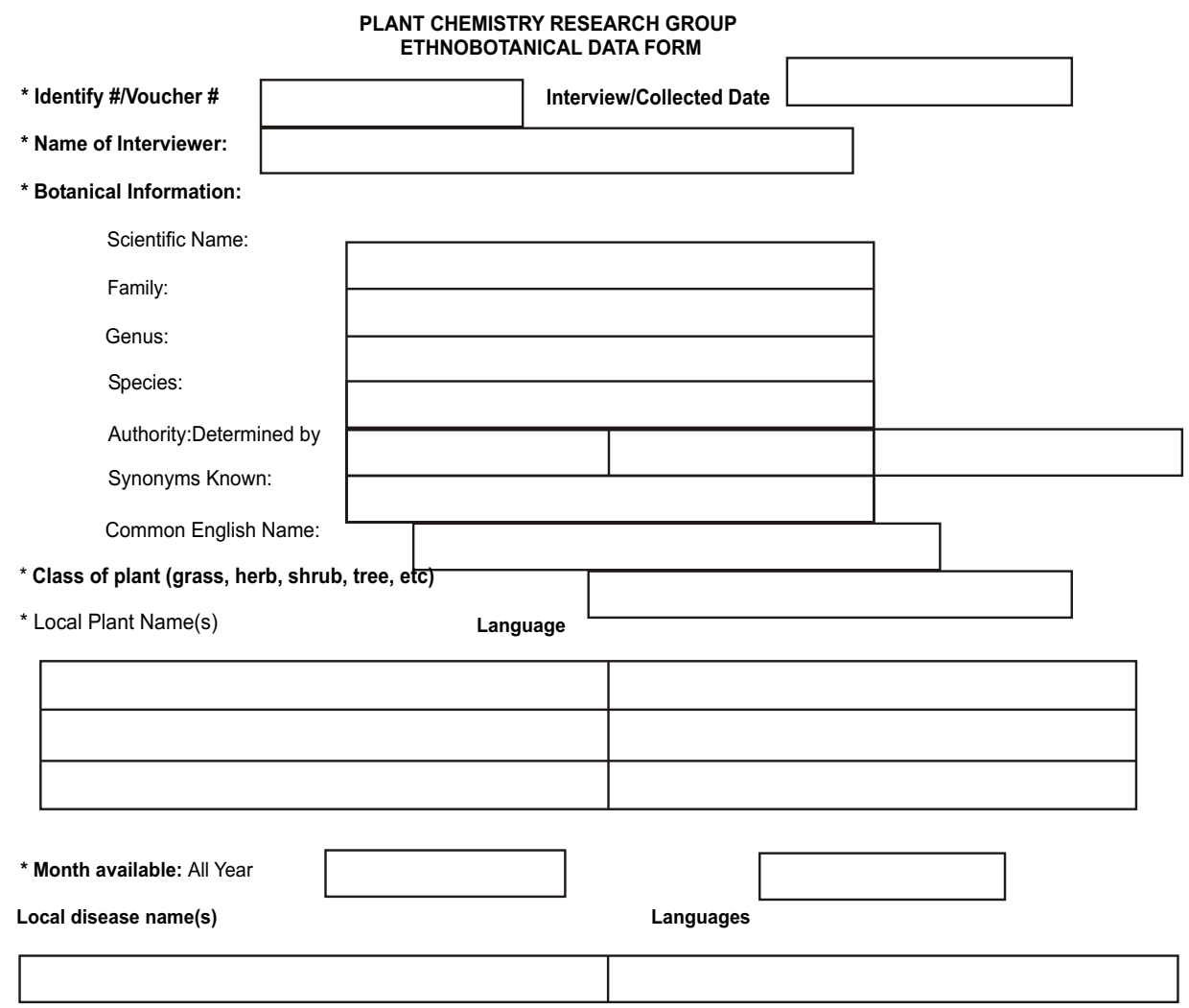

*English Name of the disease

* Non-medical ethno-botanical uses of plant (e.g. food, fiber, ritual and spiritual uses:

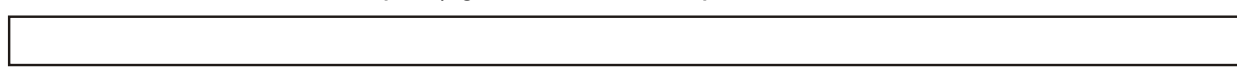

* Ethnopharmacological preparation:

Specific part(s) of plant use: (shoot \{heartwood/stem bark\}, root \{heartwood/stem bark\}leaves. Seed and fruit)

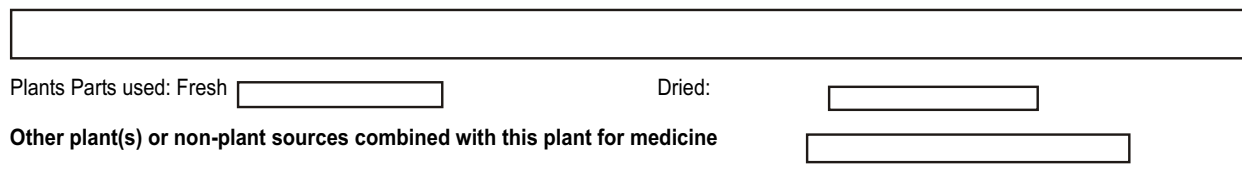

* Mode of preparation (including volume or weight of the plant materials) state the technique of collection and the preferred collection time of year (or day/night): 
* Mode of application:

How the medicine is taken (e.g oral, topical, inhalant, suppository)

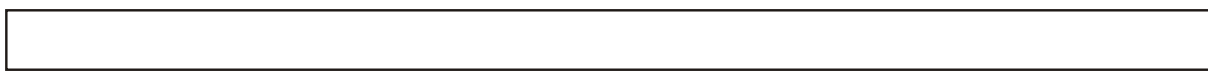

${ }^{*}$ Amount of medicine used per dose (e.g. grams, milliliters, etc)

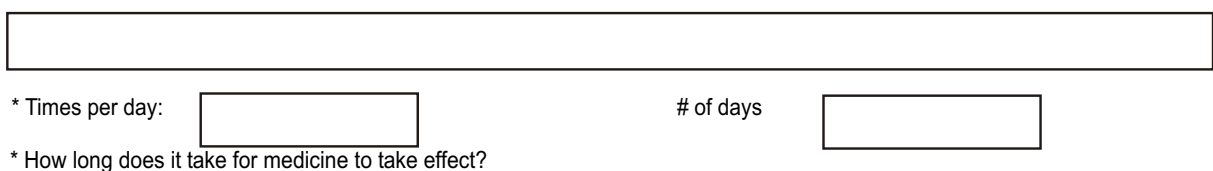

* How long does it take for medicine to take effect?

*Side effects or toxicity: Yes $\square$ No $\square$ If Yes, describe

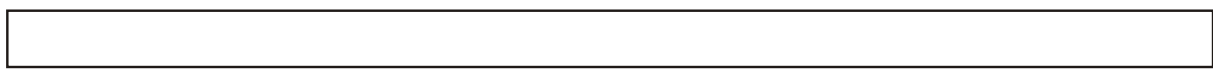

${ }^{*}$ Any dietary modifications to treat disease: Yes $\square$ No $\square \quad$ N

* Summary of Ethnomedical uses from literature:

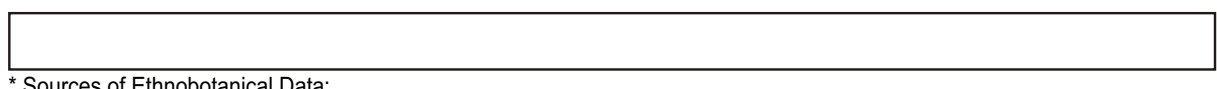

Name of Interviewer:
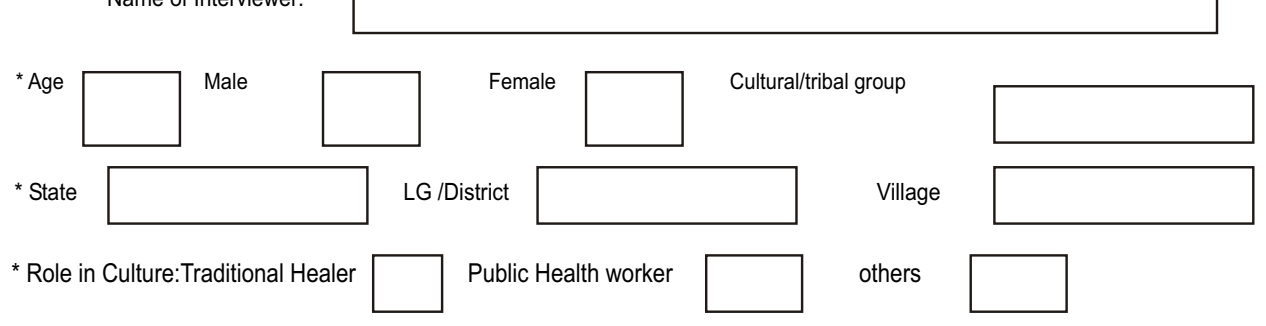


\section{Discussion}

The method employed for this study had been used successfully for many years in Haiti [32] and among the Baka Pygmies of Cameroon [12]. In the ethnobotanical study of Mexican plants, Jimenez-Arellanes and co-workers in 2003 classified symptoms of tuberculosis as fever, cough, blood in the sputum and other pulmonary diseases [17].

In terms of the number of citations for treating respiratory diseases (Table 3 ), tuberculosis is the most important disease, followed by cough. Asthma and hemoptysis occupy third and fourth positions respectively. From the survey of literature of medicinal plants for treating human diseases, the respiratory ailments generally tend to be the most important disease to be treated with herbal medicine in the recent years $[13,15,17]$. This may be due to the co-infection of HIV/AIDS with respiratory diseases particularly TB. Table 3 shows that tuberculosis, cough, asthma, hemoptysis and bronchitis were the most frequently cited by the medicinal healers of Niger State. Table 2 revealed that the majority of the identified plants and recipes were used widely judging from the respective number of citations for asthma (3); cough (3); hemoptysis (3) and tuberculosis (4). This may be due to the general interactions at the societal levels particularly the interactions at the professional level of the traditional medicine practitioners. However, some herbal remedies are made up of animal parts such as the lungs of rabbit, Poelagus marjorita; head and body of lizard, Agama agama, insect parts, and oyster shell (Ostrea spp) were reported as common ingredients in the traditional treatment of TB and other respiratory diseases.

Although, all the sample population for this study comprised of all respondents interviewed, for various reasons, it was not possible to obtain equal number of plant materials from all of them. The correctness of the information from respondents could be checked from the frequency of use by different healers across the zones of the state and also from the medicinal uses reported in the literature, which is often corroborated by values of the performance index of medicinal plant. It is noteworthy that the plant species must frequently used for a specific respiratory ailment (with higher value for $I p$ ) are known in the literature to possess biologically active compounds. The following are eleven of such plants:

(1) Abrus precatorius (Papilionaceae) was recorded among the three major ethnic groups in Niger state for use against bronchitis, cough and tuberculosis $(I p>1)$ similar to the works of Disengomoka (1983) and Hedberg (1982) and their coworkers who reported the use of its root and leaves for treating these ailments [ $[15$, 17]. The active compounds associated with these activities isolated from the aerial parts of this plant are abruquinone B and abruquinone G. These particularly abruquinone B exhibited antitubercular, antiplasmodial and cytotoxic activities 
[33].

(2) Aframomum melegueta (Zingiberaceae) was also recorded for treating bronchitis, cold, expectorant and fever (Ip>1). Similarly, Gill (1992) and Betti (2004) reported the usefulness of this plant for the treatment tuberculosis, cough and chest congestion $[12,22]$. Galal (1996) reported the actimycobacterial activity of 6-paradol and 6-shogaol isolated from the seeds of this plant at 10-15ug/ml [34].

(3) Allium ascalonicum (Lamiaceae) is mentioned to be frequently used against asthma, cough and hemoptysis $(I p>1)$. It was reported to be used as stimulant, expectorant and diuretic [3] and these activities are attributed to the presence of sulphur compounds [22].

(4) Allium sativum (Lamiaceae) is also frequently used for the treatment against asthma, cough and tuberculosis $(I p>1)$. Etkin (1981) reported the use of the decoction of the bulb for the asthma, fever, bronchial expectorant [6]. Delaha and Garagusi (1985) and Jain (1999) reported anti-TB activity of the isolated flavonoids namely: licoisoflavone $(50 \mathrm{ug} / \mathrm{ml})$ and allicin $(1: 60)[35,36]$.

(5) Detarium microcarpum (Caesalpiniaceae) is frequently used against asthma, cold and hemoptysis $(I p>1)$. The decoction of the root and leaves was earlier reported to be used for curing wounds, ulcers and fresh cuts due to the presence of detaric acid [3].

(6) Elaeis guineensis (Palmae) is much used against asthma, bronchitis, chest pain and tuberculosis $(I p>1)$. Most tropical forest plants are used in treating tropical diseases and the seeds or oils of this plant are often associated other plants [12].

(7) Piliostigma thonningii (Caesalpiniaceae) is frequently mentioned for treating chest pain, hemoptysis and sore throat (Ip>1). Similarly, decoction of the fresh young leaves, dried stem bark and root bark were earlier reported to have been used for the treatment of chest complaint, cough, fever and expectorant[3,22].

(8) Sterculia setigera (Sterculiaceae) is commonly used in folk medicine to treat different respiratory diseases such as asthma, bronchitis, cough and hemoptysis $($ Ip $>1)$.

(9) Terminalia avicennioides (Combretaceae) was recorded for use against bronchitis, hemoptysis and tuberculosis $(I p>1)$. A member of this genus exhibited a broad spectrum of antimicrobial activity, particularly significant against $S$. aureus, $M$. luteus, P. vulgaris, $P$. aeruginosa and E. coli [37]. The aqueous extract of the stem bark and roots have been reported to be active against Mycobacterium phlei [38]. Similarly, Katerere (2003) and his group isolated imberbic acid with potent activity against Mycobacterium fortuitum and S. aureus from the stem bark of Terminalia spp. [39].

(10) Vitellaria paradoxa (Sapotaceae) is the commonest for treating asthma, expectorant, tuberculosis and whooping cough (Ip>1). Gill (1992) earlier reported the use of the decoction of seeds for treating catarrh condition and nasal decongestion [22]. 
(11) Entada africana (Mimosaceae) was found to be useful for treating bronchitis $(I p>1)$ and whooping cough $(I p>2)$. Previous studies showed that this plant displayed significant antimicrobial activity by Oyagade (1994) [40] and the associated constituents were reported as paucine, rotenone and saponins [22].

The medicinal applications of indigenous flora for respiratory diseases as reported for Niger State, Nigeria are to a large extent similar with the earlier surveys of Nigerian medicinal plants i.e. Western Nigeria and Eastern Nigeria ethnomedicines [2, 10]; Hausa medicinal plants [6]; Bauchi State herbal plants [38]; Igede medicinal practices [41, 42]; Tiv anti-malarial medicinal plants [43] and others include Zaire medicinal plants [15]; Tanzania medicinal plants [16]; Cameroonian medicinal plants [12] and Mexican medicinal plants [17].

Survey of literature showed that some of these plants are used in other states of Nigeria for treating TB and related respiratory diseases [2, 3, 4, 6, 11, 30, 41, 42, 45]; particularly in the middle belt states of Nigeria comprising of Kwara, Kogi, Kebbi, Kaduna, Plateau, F.C.T, Benue, Nasarawa, Taraba and Adamawa. These states belong to the same vegetational zone of Savannah-Guinean forest area (inhabited by some Nigeria's minority tribes that are culturally deeply rooted in the practices of traditional medicine and farming). They share many common plants [3, 41-45].

This survey also revealed that the plants used by the indigenous peoples of Niger State for respiratory diseases have some similarities with the plants used in other parts of the Africa [5, 7, 8, 13-20, 46-49]. In this study, plant species to be noted are those more frequently used for TB and other pulmonary disorders (with higher value for $I p$ ) that are known in the literature to possess active compounds [4, 50, 51]. Literature review showed that plants with high $I p$ often have effective chemical substances. Moreover, many of the identified plants particularly those with $(I p>0)$ are known to contain terpenes, saponins, phenols, polyphenols, tannins, flavonoids and alkaloids [52-58], which are metabolites known to be responsible for the associated activities.

In the present study, the main purpose is to establish an inventory of herbal recipes and the constituent medicinal plants for TB and respiratory diseases in Niger State, which is presently lacking in literature. The crude extracts of many plant species, especially those with ethnomedical uses have been assessed for in vitro antimycobacterial properties [51, 59-64]. Therefore, the interest for this report is strengthened due to the fact that some of the medicinal plants reported in this survey were earlier shown to have exhibited significant antimycobacterial activities [51, $64,65]$. A number of plant extracts and compounds have also exhibited potent antituberculosis properties [51]. Among the plant species from other study areas that indicated such anti-TB properties include Allium sativum (Garlic), Eucalyptus spp (Eucalyptus), Ricinus communis (Castor bean), Cocos nucifora (Coconut), Eugenia $s p$ (Clove), Zingiber officinale (Ginger), Psidium guajava (Guava), Moringa 
pterygosperma (Horse raddish tree), Cola nitada (Kola), Lawsonia spp(Henna), Mangifera indica (Mango), Azadrachita indica (Neem), Carica papaya (Pawpaw) and Fagara zanthroxylodes (Prickly ash). It is hoped that these plant species would be thoroughly investigated particularly those with high performance indices $(I p>0)$, which are yet to be studied.

As the WHO increases its drug discovery efforts for new anti -tubercular drugs that can offset multidrug resistance, the indigenous knowledge from traditional medicine of the peoples of Niger State, Nigeria, becomes valuable for identifying new anti-tubercular and other anti-infective compounds.

\section{Conclusions}

Some of the herbal recipes from Niger State, Nigeria found to be widely used for the treatment of $\mathrm{TB}$ and other respiratory diseases contain plants with high performance indices. This observation is an indication of potential value of these plants as good source of 'lead' compounds for the development of new antitubercular and anti-infective drugs. In this survey, most of the identified medicinal plants with high performance indices are known for their effective properties against certain respiratory diseases, which lend credence to the ethnomedicine from the indigenous flora of Niger State. The present results are encouraging; therefore, there will be no need for any further random searching for active chemical substances to curb multidrug resistant TB and other respiratory diseases. It is suggested that further investigations involving in vitro and in vivo tests be conducted on the bioactive extracts/compounds isolated from plants with high performance indices.

\section{Acknowledgments}

We want to express our words of appreciation to all the healers (particularly Muhammad Mamman Kocita-Doko, Usman Simon Jiya Pici and Baba Alhassan Bangbara Bida for undertaking all journeys) that made this survey possible. Our profound gratitude goes to Community Leaders (especially Etsu Nupe for his royal support and encouragements) and officials of the professional herbal association for their help in establishing contact with the healers thereby removing a lot of hurdles for us. Finally, we thank these officers in the Herbaria (Muhammad Musa and Umar S Gallah of the Department of the Biological Sciences, ABU Zaria and Ibrahim Muazzam, Jemilat Ibrahim and Grace Ugbabe of NIPRD, Idu-Abuja) for identifying the plant species. We appreciate effort of Mallam Mohammed Ndagba who typeset the work. 


\section{References}

[1] Mann, J. (1992). Murder, Magic and Medicine. Oxford University Press, London, p. 35.

[2] Iwu, M.M. (1993). Handbook of African Medicinal Plants. C.R.C. Press, Florida, p. 64.

[3] Mann, A., Gbate, M. and Nda-Umar, A. (2003). Medicinal and Economic Plants of Nupeland. $1^{\text {st }}$ Ed. Jube-Evansbooks and Publications, Bida, 279 pp.

[4] Oliver-Bever, B. (1986). Medical Plants in Tropical West Africa. Cambridge University Press, Cambridge, Great Britain, 375 pp.

[5] Kerharo, J. and Adam, J.G. (1974). La Pharmacopee Sénégalaise Traditionnelle, Vigot Freres, Paris, 1011 pp.

[6] Etkin, N.L. (1981). A Hausa herbal pharmacopoeia: Biomedical evaluation of commonly used plant medicines, J. Ethnopharmacol., 4: 75-98.

[7] Ayensu, E.S. (1978). Medicinal Plants of West Africa. Reference Publications, Algonac, Michigan, $330 \mathrm{pp}$.

[8] Cunningham, A.B. (1993). African Medicinal Plants: Setting priorities at the interface between conservation and primary health care. People and Plants Working paper 1. Paris. UNESCO, pp. 1-50.

[9] Snider, D. E. T., Raviglione, M. and Kochi, A. (1994). A Global burden of tuberculosis, Tuberculosis: Pathogenesis, Protection, and Control. Barry Bloom (Ed.), ASM Press, Washingston, D.C.p. 3.

[10] WHO web sites. Links for an overview of tuberculosis worldwide.

[11] Adjanohoun, E., Ahyi, M.R.A., Ake Assi, L.,Elewude, J. A., Dramane, K., Fadoju, S. O., Gbile, Z. O., Goudole, E., Johnson, C. L. A., Keita, A., Morakinyo, O., Ojewole, J. A. O., Olatunji, A. O., Sofowora, E. A.(1991). Traditional Medicine and Pharmacopoeia. Contribution to ethnobotanical floristic studies in Western Nigeria, Pub. Organization of African Unity, Scientific Technical and Research Commission Lagos, Nigeria. 420 pp.

[12] Betti, J.L. (2004). An Ethnobbotanical Study of Medicinal Plants among the Baka Pygmies I the Dja Biosphere Reserve, Cameroon. Africa Study Monograph, 25: 1-27.

[13] Chhabra, S.C., Mahunnah, R.L.A. and Mshiu, E.N. (1990). Plants used in traditional medicine in Eastern Tanzania IV. Angiosperms (Mimosaceae to Papilionaceae). $J$. Ethnopharmacol., 29:295 323.

[14] Diafouka, A. J. P. (1997). Analyse des usages des plantes médicinales dans 4 régions de Congo-Brazzaville. Thèse de doctorat, Université libre de Bruxelles, Faculté des Sciences, Laboratoire de Botanique Systématique et de Phytosociologie, 431pp.

[15] Disengomoka, I., Delaveau, P. (1983). Medicinal Plants used for child's respiratory diseases in Zaire, Part I., J. Ethnopharmacol., 8: 257-263.

[16] Hedberg, I., Hedberg, O., Madati, P., Mshigeni, K.E., Mshiu, E.N.and Samuelsson, G.(1982). Inventory of plants used in traditional medicine in Tanzania. I. Plants of the families Acanthaceae - Cucurbitaceae. J. Ethnopharmacol., 6: 29 - 60.

[17] Jimenez-Arellanes, A., Meckes, M., Ramirez, R., Tores, J.and Luna-Herrera, J.(2003). Activity against multidrug-resistant Mycobacterium tuberculosis in Mexican plants used to treat respiratory diseases. Phytotherapy Research 17: 903-908.

[18] John, E. (1985). A barefoot doctor's Manual: Practical Clines Medicine and Health. Gramercy Publishing Company, New York, $1001 \mathrm{pp}$.

[19] Pamplona-Roger, G. D. (ed.) (1999). Encyclopedia of Medicinal Plants. Vol.1 Education and Health Library, $998 \mathrm{pp}$.

[20] Wome, B. (1985). Recherches ethnopharmacognosiques sur les plantes médicinales utilisées en médecine traditionnelle à Kisangani (Haut-Zaïre). Thèse de doctorat, Université libre de Bruxelles, Fac. Sc., $561 \mathrm{pp}$.

[21] Dalziel, J.M. (1937).Useful Plants of West Tropical Africa.Crown Agents, London, $296 \mathrm{pp}$.

[22] Gill, L.S.(1992). Ethnomedicinal uses of plants in Nigeria. Uniben press, Benin City, 
Nigeria, $276 \mathrm{pp}$.

[23] Ainslie, J.R.(1937). A list of Plants Used in Native Medicine in Nigeria. Oxford University Press, London, p. 54.

[24] Mann, A., Abalaka, M. E. and Garba, S.A. (1997). The antimicrobial activity of the leaf extracts of Calotropis procera. Biomedical Letters; 55:205-210.

[25] Mann, A. Suleiman, M. A. T. (1998). Cytotoxic and antimicrobial activities of the stem bark extract of Sclerocarya birrea. J. Chem. Soc. Nigeria. 23:16-18.

[26] Mann, A. and Okwute, S. K. (1999). Phytochemical and biological Screenings of Commiphora kerstingii Engl. (Burseraceae). Zuma J. Pure \& Appl. Sci. 2(1): 69-72.

[27] Malcolm, S.A and Sofowora, E.A. (1969). Antimicrobial activities of selected Nigerian Folk Remedies and their Constituent plants. Lloydia, 32:512-517.

[28] Hostettman, K. (1998). Strategy for the Biological and Chemical Evaluation of Plant Extracts "Invited lecture presented at the Internal Conference on biodiversity and Bioresource: Conservation and utilization, 23-27 November, 1997, Phuket, Thailand.

[29] Iwu, M.M. (1994). African Medicinal Plant in the Search for new drugs based on ethnobotical leads. In: (D.J. Chadwick and J. Marsh, eds.) Ethnobotany and the search for new drugs. Ciba Foundation Symposium, Wiley, Chichester, 185: 116-129.

[30] Patel, M. B.and Rowson, J. M. (1964). Investigation of Certain Nigerian Medicinal Plants. Part-I. Preliminary Pharmacological and Phytochemical Screenings for Cardiac activity. Planta Medica 12: 33-42.

[31] Khafagi, I. L.and Dewedar, A. (2000).The efficiecy of random versus ethno-directed research in the evaluation of Sinai medicinal plants for bioactive compounds. J.Ethnopharmacol.71:365-376.

[32] Robineau, L. (1991). Vers une pharmacopée caraibe. Sse'minaire Tramil 4, Enda-caraibe et UNAH, Santo Domingo.

[33] Limmatvapirat, C., Sirisopanaporn, S.and Kittakoop, P. (2004). Antitubercular and antiplasmodial constituents of Abrus precatorius.Planta Medica 70: 276-278.

[34] Galal, A.M. (1996). Antimicrobial activity of 6-paradol and related compounds. $J$. Pharmmacog., 34: 64-69.

[35] Delaha, E.C., Garagus, V.F. (1985). Inhibition of Mycobacteria by garlic extract (Allium Sativum). Antimicrobial Agents Chemotherapy, 27: 485-486.

[36] Jain, R.C. (1999). Antitubercular activity of garlic oil [Letter]. Indian Journal Pathology Microbiolology 41:31.

[37] Oyagade,J.O. (1997). Antimicrobial efficacy of stem bark extracts of Terminalia schimperiana (Hochst). Bioscience Research Communications, 30: 143-149.

[38] Adamu, H.M., Abayeh, O.J., Agbo, M.O., Abdullahi, A.L., Uba, A., Dukku, H.U., Wufem, B.M. (2005). An ethnobotanical survey of Bauchi State herbal plants and their antimicrobial activity. J. Ethnopharmacol.,99: 1-4.

[39] Katerere, D. R. P., Gray, A. I., Nash, R. J. and Waigh, R. D. (2003). Antimicrobial activity of pentacyclic triterpenes isolated from African Combretaceae. Phytochemistry, 63: 81-89.

[40] Oyagade, J. O. (1994). The antimicrobial efficacy of stem bark extracts of two Nigerian Medicinal Plants, Terminalia glauceccens Planch and Entada africana Guill and Perr. Ph.D. Thesis University of Ilorin, Nigeria.

[41] Igoli, J.O., Igwue, I.C.and Igoli, N.P. (2003). Traditional Medicinal Practices among the Igede People of Nigeria. Journal of Herbs, Spices and Medicinal Plants. 10: 1-10.

[42] Igoli, J.O., Ogaji, O.G., Tor-Anyiin, T.A. and Igoli, N.P. (2005). Traditional medicine practice amongst the Igede people of Nigeria, Part II. African Journal of Traditional, Complementary and Alternative Medicines. 2:134-152.

[43] Tor-anyiin, T.A., Shaato, R.and Oluma, H.O.A. (2003). Ethnobotanical Survey of anti malarial Medicinal Plants amongst the Tiv people of Nigeria. Journal of Herbs, Spices \& 
Medicinal Plants. 10: 61-74.

[44] Oliver-Bever, B. (1983). Medicinal Plants in tropical West Africa III. Anti-infection therapy with higher plants. J. Ethnopharmcol., 9:1-83.

[45] Hussain, H.S.N., Karatela Y.Y. (1989). Traditional medicinal plants used by Hausa tribe of Kano State of Nigeria International Journal Crude Drug Research 27: 211-216.

[46] Kokwaro, J.O.(1976). Medicinal Plants of East Africa. East African Literature Bureau, Nairobi, Kenya, 368 pp.

[47] Boulos, L . (1983). Medicinal Plants of North Africa. Reference Publications, Inc. Algoac, Michigan, 286 pp.

[48] Boiteau, P. (1986). Médecine traditionnelle et pharmacopée.Précis de matière médicale malgache.Agence de coopération culturelle et technique,A.C.C.T.ed., Paris, $141 \mathrm{pp}$.

[49] Msonthi, J. D. (1994). Research in to medicinal plants of Malawi.Vol. 1 \& 2. (J. H. Seyani \& A. C. Chicuni (ed.) National herbarium and Botanic Gardens of Malawi, Zomba, Malawi. $1514 \mathrm{pp}$.

[50] Copp, B.R. (2003). The Review of Natural Products with antimicrobacterial activity. Nat. Prod. Report, 20: 535-557.

[51] Newton, S.M., Lau, C.and Wright, C. W. (2000). A review of natural antimycobacterial products. Phytotherapy Research, 14:303-322.

[52] Cantrell, L., Franblau, S.G and Fischer, N.H. (2001). Antimycobacterial plant terpenoids. Planta Medica 67: 685-694.

[53] Koysomboon, S., Altena, I.V., Kato, S.and Chantrapromma, K. (2006). Antimycobacterial flavonoids from Derris indica, Phytochemistry 67: 1034-1040.

[54] Lall, N., Hussein, A.A.and Meyer, J.J.M. (2006). Antiviral and antituberculosis activity of Helichrysum melanacme constituents. Fitoterapia 77:230-232.

[55] O'Donnell, G., Bucar, F.and Gibbons, S. (2006). Phytochemistry and antimycobacterial activity of Chlorophytum inornatum, Phytochemistry 67: 178-182.

[56] Wachter, G. A., Valcic, S., Flagg, M. L., Franzblau, S.G., Suarez, E; Mantenegro, G. and Timmermann, B. N. (1999). Antitubercular activity of pentacyclic triterperiods from plants of Argentina and Chile. Phytomedicine 6: 341-345.

[57] Wachter, G. A., Valcic, S., Franzblau, S.G., Suarez, E.and Timmermann, B. N. (2001). Antitubercular activity of triterpenoids from Lippia turbinatta., J. Nat. Prod., 64:37-41.

[58] Wube, A.A., Bucar, F., Gibbons, S. and Asres, K. (2005). Sesquiterpenes from Warburgia ugandensis and their antimycobacterial activity. Phytochemistry, 66: 2309-2315.

[59] Fitzpatrick, F.K. (1954) . Plant Substances active against Mycobaterium tuberculosis. Antibiotic and Chemotherapy 4: 528-536.

[60] Katerere, D.R., Gray A.I., Nash, R.J. and Waigh, R.D. (2003). Antimicrobial activity of pentacyclic triterpenes isolated from African Combretaceae. Phytochemistry 63: 81-88.

[61] Kirdmanee, C., Kamolcchanok, R. and Palittapongarnpim, P. (2004). Efficacy of Thai Traditional Herbal Plants for Antituberculosis. National Center for Genetic Engineering and Biotechnology, P-Plant-06. Thailand.

[62] McCutcheon, A.R., Stokes, R.W., Thorsonn, L.M., Ellis, S.M., Hancock, R.E.W., Towers, G.H.N. (1997). Anti-Mycobacterial Screening of British Columbian Medicinal Plants. Pharmaceutical Biology 35: 77-83.

[63] Tosun, F., Kizilay, C.A., Sener, B., Vural, M. and Palittapongarnpim, P. (2004). Antimycobacterial Screening of some Turkish plants. J. Ethnopharmacol., 95: 273-275.

[64] Uba, A., Ibrahim, K., Agbo, E. B. and Makinde, A. A. (2003). In vitro inhibition of Mycobacterium smegmatis ATCC607 and a clinical isolate of Mycobacterium tuberculosis by some Nigerian Medicinal Plants. Science Forum: Journal Pure and Applied Science, 6: 226-231.

[65] Ashidi, J.S., Ayodele, A.E. and Gbile, Z.O. (1997). Ethnobotanical Studies of Antituberculosis plants in Egbado (Nigeria). Indigenous Knowledge ad Development Monitor, 5: Sept. 1997. 